\title{
A Thorough Examination of Morning Activity Patterns in Adults with Arthritis and Healthy Controls Using Actigraphy Data
}

\author{
Alison Keogh ${ }^{a, b} \quad$ Niladri Sett ${ }^{a, c}$ Seamas Donnelly ${ }^{d}$ Ronan Mullan ${ }^{d}$ \\ Diana Gheta ${ }^{e}$ Martina Maher-Donnelly ${ }^{d}$ Vittorio Illiano ${ }^{f}$ \\ Francesc Calvo $^{f}$ Jonas F. Dorn ${ }^{f}$ Brian Mac Namee ${ }^{a, c} \quad$ Brian Caulfielda, $^{a}$ \\ ${ }^{a}$ Insight Centre for Data Analytics, University College Dublin, Dublin, Ireland; ${ }^{b}$ School \\ of Public Health, Physiotherapy and Sports Science, University College Dublin, Dublin, \\ Ireland; ' School of Computer Science, University College Dublin, Dublin, Ireland; dSchool of

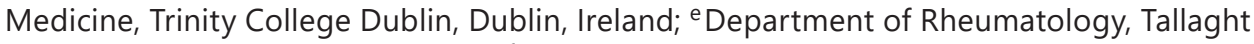 \\ University Hospital, Dublin, Ireland; ' Data and Digital, Novartis, Basel, Switzerland
}

\section{Keywords}

Actigraphy · Arthritis · Remote monitoring · Digital biomarkers

\section{Abstract}

Background: Wearable sensors allow researchers to remotely capture digital health data, including physical activity, which may identify digital biomarkers to differentiate healthy and clinical cohorts. To date, research has focused on high-level data (e.g., overall step counts) which may limit our insights to whether people move differently, rather than how they move differently. Objective: This study therefore aimed to use actigraphy data to thoroughly examine activity patterns during the first hours following waking in arthritis patients $(n=45)$ and healthy controls $(n=30)$. Methods: Participants wore an Actigraph GT9X Link for 28 days. Activity counts were analysed and compared over varying epochs, ranging from 15 min to $4 \mathrm{~h}$, starting with waking in the morning. The sum, and a measure of rate of change of cumulative activity in the period immediately after waking (area under the curve [AUC]) for each time period, was calculated for each participant, each day, and individual and group means were calculated. Two-tailed independent $t$ tests determined differences between the groups. $\boldsymbol{R e}$ sults: No differences were seen for summed activity counts across any time period studied. However, differences were noted in the AUC analysis for the discrete measures of relative activity. Specifically, within the first 15, 30, 45, and 60 min following waking, the AUC for activity counts was significantly higher in arthritis patients compared to controls, particularly at the 30 min period ( $t=-4.24, p=0.0002)$. Thus, while both cohorts moved the same amount, the way in which they moved was different. Conclusion: This study is the first to show that a 
detailed analysis of actigraphy variables could identify activity pattern changes associated with arthritis, where the high-level daily summaries did not. Results suggest discrete variables derived from raw data may be useful to help identify clinical cohorts and should be explored further to determine if they may be effective clinical biomarkers.

(C) 2020 The Author(s)

Published by S. Karger AG, Basel

\section{Introduction}

The sustained growth of chronic disease places significant burden on global health resources [1], resulting in the need to identify and develop more effective forms of treatment and prevention strategies. In response, the development of biomarkers, defined as "characteristics that are objectively measured and evaluated as indicators of normal biologic, pathologic, or biological responses to a therapeutic intervention" [2], has demonstrated promise as an effective way to help capture disease-specific, clinically relevant changes in cohorts over time. More recently, advances in mobile and digital technology have led to the creation of a new class of biomarkers, digital biomarkers, which are prognostic or diagnostic measurements of health collected using digital means [2].

Digital biomarkers offer researchers and clinicians access to large volumes of objective data that can be captured relatively passively within the context of free-living "normal" conditions $[3,4]$. As the algorithms and hardware of digital devices improve, so too does their potential to offer more nuanced and comprehensive insights into the characteristics of diseases [5]. This may also be beneficial to patient cohorts, through the identification of clinically meaningful parameters in their specific conditions. Indeed, identifying information that is important to patients is a primary goal of future drug and treatment developments [6]. However, significant challenges remain, including: how best to use the data derived from these devices [2, 4], ensuring reliability and reproducibility of results [2, 3, 7], an imposed reliance on the proprietary algorithms of individual devices, and the selection of clinically meaningful endpoints. Thus, identifying specific digital biomarkers that are best able to differentiate between healthy and clinical cohorts is a critical first step in this process. This may support the development of new models for clinical trials to subsequently test the efficacy of these markers. Once achieved, the next step is to understand how well these biomarkers capture change in patient status over time, or as a result of therapeutic intervention.

Physical activity (PA) is one area of healthcare where there has been an explosion of digital data collection over recent years, as advances in wearable sensor technology have led to the wide availability of both medical and consumer-based devices. Recent research suggests that digital biomarkers may be identified using devices such as mobile phones; however, to date, they have relied on specific tasks rather than PA in general [8]. PA is a critical determinant of health and thus is a key outcome for a range of chronic illnesses including chronic musculoskeletal conditions, such as arthritis. The objective measurement of PA was previously limited by a reliance on self-report or in-laboratory data [9-11]; however, wearable sensors now allow researchers to remotely and accurately monitor daily movement patterns over time using small, unobtrusive devices $[9,12]$. Indeed, this area has expanded rapidly over the past decade as advances in usability and device specifications have supported participant adherence [13]. These improvements therefore offer valuable opportunities to researchers to capture digital health data which may be used as a digital biomarker. Previous research has used actigraphy to explore PA patterns in specific disease cohorts [10,14-18]. Establishing activity differences between healthy controls and arthritic patients though has been less clear, with some studies identifying differences $[10,15,17]$ while others suggest that none or minimal exist $[16,18]$. However, these results have been based on the evaluation

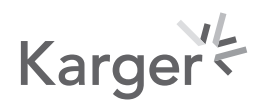


of summary-level data (e.g., overall step counts or daily activity) [19], which may limit our insights to simply whether people move differently, rather than how they move differently. Deriving a deeper understanding into how and when people move may provide researchers with a more valuable and sensitive insights into how conditions such as arthritis impact people's behaviour and daily functional tasks.

To identify potential digital biomarkers, it may be a valuable first step to explore whether certain disease characteristics can be identified through actigraphy data. Specifically for arthritis, morning stiffness is a commonly reported symptom [20,21]. How, and indeed if, disease-specific symptoms such as this impact a person's activity patterns is, as yet, unknown beyond qualitative [22] or summary-level data [10,23]. Recent research has suggested that there may be a difference in the morning activity patterns between arthritis patients and healthy controls; however, the collected actigraphy data was listed as a percentage of the overall activity during the day and was measured over multiple hours up to midday, therefore it is not specific to the morning alone [10]. Thus, a deeper understanding as to how people with chronic conditions move, and what we can learn from this, requires researchers to derive more detailed and discrete variables from the minute-level actigraphy data that they collect, in order to firstly determine whether they are more sensitive to differences than summarylevel assessments such as daily activity counts. This level of data processing has not been previously undertaken, therefore it is unknown whether more detailed time-driven analysis of actigraphy data offers any value in enhancing our understanding of conditions such as arthritis, and subsequently whether these variables may be utilised in future clinical trials as digital biomarkers. The aim of this study was therefore to use actigraphy data to perform a thorough examination of activity patterns during this morning period in patients with arthritis and healthy controls. Groups' actigraphy variables were analysed and compared over varying epochs, ranging from 15 min to $4 \mathrm{~h}$, starting with waking in the morning.

\section{Materials and Methods}

Ethical approval for this study was granted by the Human Ethics Board of the local research institution. The participants and data within this study are the same as those previously reported elsewhere [24].

\section{Study Design and Participants}

This was a non-interventional 4-week longitudinal study that took place between March and September 2016. Healthy volunteers $(n=30)$ were recruited through convenience sampling, using notices placed in local environments. Participants with arthritis were recruited through Rheumatology Department at Tallaght Hospital, Dublin, Ireland $(n=45)$. Arthritis participants were required to fulfil disease-specific classification criteria as determined by the rheumatologist within the hospital clinic (Table 1). Prior to inclusion within the study, all participants underwent a clinical assessment and provided written informed consent. As this was an exploratory study, no formal sample size calculation was performed. Basic participant demographic information (i.e., height, weight, clinical characteristics) were collected at baseline.

\section{Procedures}

The participants were provided with an Actigraph GT9X Link wrist-worn sensor which they were asked to wear continuously (except when showering and charging) for the duration of the study ( 4 weeks). The participants were required to charge the monitors but otherwise did not have to engage with the devices. Activity feedback from the device was disabled so as

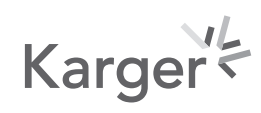


Table 1. Inclusion and exclusion criteria

\author{
Healthy participants \\ Inclusion criteria \\ Adult (aged 18-80 years) \\ Independent in daily activities \\ No history of diagnosis or treatment of arthritis \\ Not currently receiving active treatments for disabling illness
}

\title{
Arthritis participants
}

Inclusion/classification criteria

Adult (aged 18-80 years)

Attending the Rheumatology Department of Tallaght Hospital with one of the following: rheumatoid arthritis (RA), osteoarthritis (OA), psoriatic arthritis (PsA), ankylosing spondylitis (according to the below criteria)

- Rheumatoid arthritis - RA 2010 ACR/EULAR criteria [19]

- Osteoarthritis - ACR classification OA of one or more hip or knee [22]

- Psoriatic arthritis - Psoriatic arthritis CASPAR classification criteria [20]

- Ankylosing spondylitis - Ankylosing spondylitis ASAS 2012 criteria for axial spondyloarthopathy [21]

Exclusion criteria

Additional non-arthritic conditions that may impact the participant's physical activity

No capacity to give informed consent

Pregnant at the time of recruitment

not to influence the participants' normal daily activities. As reported elsewhere, a smartphone app (CentrosHealth, Boston, MA, USA) was used to collect daily patient-reported outcomes (PROs) regarding the degree of pain and stiffness perceived, using 8 questions [21].

\section{Data Processing and Analysis}

Actigraph raw accelerometer data gives measurements of the movements at the wrist in the $x, y$, and $z$ axes, indicating the activity levels of the participant. Raw accelerometer data was collected at $30 \mathrm{~Hz}$. Full processing details of this raw data are reported elsewhere [25]. To reduce variability, the raw data was re-calibrated using a method adapted from van Hees et al. [26]. The calibrated acceleration data was imported back into the ActiLife software to get the corrected 60-s epoch activity counts for the $x, y$, and $z$ axes. To facilitate sleep detection, and for more convenient visualisation, study days were converted to data analysis days (DADs). A DAD started at 18:00:033 and ended on 18:00:000 the following day. The day on which recording starts is set to DAD 0 , so that DAD 1 is the first day within the study with 24 $\mathrm{h}$ of data (assuming full compliance; all deployments were completed before 18:00). The 60-s epochs were then used to derive additional actigraphy variables within the DAD.

Each 60-s epoch of activity data was classified as an epoch during which the Actigraph was worn or not worn. Activity counts for each minute over the minute period following waking were extracted for each DAD for each participant. Sleep was identified using both Cole-Kripke and Tudor-Locke algorithms as described elsewhere [22]. Activity counts were calculated by a proprietary algorithm for wrist-worn devices used in the ActiLife software that first applied a band-pass filter to the sensor data, eliminating both very slow and very rapid changes in acceleration, after which each value above a certain threshold is counted. A modified Choi algorithm was used to detect non-wear [22]. A person was included in the analysis if at least $80 \%$ of the total number of analysis days $(n=28)$ were available in the data.

Cumulative activity counts for successive 60-s epochs were plotted for each of the following time periods following waking: $15,30,45,60,120,240$, and 1,440 min. The sum, and area under the curve (AUC), for activity counts for each of these time periods was calcu-

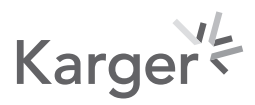


Table 2. Baseline demographic details of the participants

\begin{tabular}{|c|c|c|c|c|}
\hline & \multirow{2}{*}{$\begin{array}{l}\text { Healthy } \\
\text { participants } \\
(n=15)\end{array}$} & \multicolumn{3}{|l|}{ Arthritic participants } \\
\hline & & $\mathrm{OA}(n=10)$ & $\mathrm{RA}(n=18)$ & PsA $(n=2)$ \\
\hline \multicolumn{5}{|l|}{ Sex } \\
\hline Male & $5(33.3)$ & $4(40)$ & $5(18)$ & $0(0)$ \\
\hline Female & $10(66.6)$ & $6(60)$ & $13(72)$ & $2(100)$ \\
\hline Mean age $\pm S D$, years & $48.0 \pm 48.6(31-71)$ & $60.7 \pm 4.5(55-70)$ & $50.7 \pm 11.4(33-75)$ & $32 ; 63$ \\
\hline Body mass index \pm SD & $22.9 \pm 3.0(18.6-29.1)$ & $27.9 \pm 5.7(19.0-39.7)$ & $28.1 \pm 4.85(21.1-38.5)$ & $29.4 ; 29.4$ \\
\hline Disease duration \pm SD, days & $\mathrm{N} / \mathrm{A}$ & $1,936 \pm 2,531(216-7,407)$ & $3,246 \pm 3703(89-12,181)$ & 1,$809 ; \mathrm{N} / \mathrm{R}$ \\
\hline \multirow[t]{4}{*}{ Disease classification } & $\mathrm{N} / \mathrm{A}$ & 4 minimal $^{\mathrm{a}}$ & 3 remission $^{\mathrm{b}}$ & 1 remission $^{\mathrm{b}}$ \\
\hline & & 4 mild & 6 low & 1 low \\
\hline & & 2 moderate & 6 moderate & \\
\hline & & & 2 high & \\
\hline Mean SF-36 baseline score ${ }^{\mathrm{C}} \pm$ SD & $\mathrm{N} / \mathrm{A}$ & & & \\
\hline Physical component & $56.3 \pm 3.3(50.4-61.9)$ & $36.0 \pm 11.3(18.7-54.9)$ & $38.0 \pm 8.8(25.7-57.1)$ & $\mathrm{N} / \mathrm{R}$ \\
\hline Mental component & $53.9 \pm 5.0(45.3-61.8)$ & $50.2 \pm 9.5(39.0-62.5)$ & $51.8 \pm 7.6(34.9-64.0)$ & $\mathrm{N} / \mathrm{R}$ \\
\hline
\end{tabular}

Values are given as $n(\%)$ or as mean \pm SD (range). ${ }^{\text {a }}$ Using Kellegren and Lawrence classification [25]; ${ }^{\mathrm{b}}$ calculated using the 28 joint Disease Activity Score [26]; ${ }^{\mathrm{c}}$ no difference between baseline and exit scores using Wilcoxon sign rank test. OA, osteoarthritis; RA, rheumatoid arthritis; PsA, psoriatic arthritis. N/R, not reported; N/A, not applicable.

lated for each participant, for each day, and individual and group means for each of the two dependent variables were calculated. The purpose of the AUC analysis was to establish the rate of change of activity. The percentage of time spent in each of the following activity categories was calculated for the daily summary data according to the following thresholds: $0-1,800$ inactive, 1,801-2,690 light, 2,691-6,166 moderate, $>6,166$ vigorous. A $\chi^{2}$ test analysed the differences between both groups for each of the activity categories. Two-tailed independent $t$ tests were performed to determine differences between patient and control groups for: (1) both variables over the different periods of time, and (2) for the differences between patient and control groups for the PROs.

The relationship between the daily aggregates of morning activity and PROs was tested by fitting a mixed-effects model with random intercept, where the dependent variable was the PRO, the independent variable was the activity aggregates, and the random intercept was a patient-wise offset. This model assigned a different intercept to each patient, while the same slope was used for all participants. The PROs were tested one by one, and the same for the activity aggregate.

\section{Results}

Participant demographics are outlined within Table 2. The participants wore the sensors $91.5 \%$ of the time on average [24]. Of those recruited, 43 (95.6\%), reached the minimum threshold of $80 \%$ of the total available analysis days. The two participants who did not reach this threshold were from the arthritis cohort.

No differences were seen between healthy controls and arthritic patients across for summed activity counts for any of the time periods studied, ranging from 15 min following waking to a full day (Table 3). Limited differences were noted between the groups for the time spent in each of the activity categories, with differences $(p<0.05)$ noted for vigorous activity at both 120 and $240 \mathrm{~min}$ (Fig. 1). However, differences between the cohorts were noted in the AUC analysis for the discrete measures of relative activity (Fig. 2). Specifically, within the first $15,30,45$, and 60 min following waking, the AUC for activity counts was significantly higher in patients with arthritis compared to controls (Table 3). This result was particularly 
Table 3. Differences in activity counts ${ }^{\mathrm{a}}$ between healthy controls and arthritis patients

\begin{tabular}{lccrc}
\hline Dependant variable & Healthy controls & Arthritis patients & $t$ test & $p$ value \\
\hline Average daily activity counts & & & & \\
Time following waking & & & & \\
15 min & $390,64.2(9,570.17)$ & $42,895.5(12,568.1)$ & -0.96 & 0.34 \\
30 min & $86,338.6(20,113.9)$ & $81,937.6(21,053.8)$ & 0.60 & 0.56 \\
45 min & $135,363.0(30,286.5)$ & $121,423.0(31,688.4)$ & 1.24 & 0.22 \\
60 min & $184,561.0(41,770.7)$ & $163,870.0(47,302.2)$ & 1.26 & 0.22 \\
120 min & $382,895.0(104,630.0)$ & $358,015.0(98,553.7)$ & 0.66 & 0.52 \\
240 min & $748,328.0(199,125.0)$ & $738,955.0(180,628)$ & 0.13 & 0.90 \\
1,440 min & $2,896,070.0(463,906.0)$ & $2,653,530.0(476,761.0)$ & 1.08 & 0.30 \\
\hline AUC activity counts & & & & \\
Time following waking & $7.84(0.78)$ & & & \\
15 min & $14.73(0.91)$ & $7.52(0.52)$ & 1.32 & 0.20 \\
30 min & $22.00(1.12)$ & $23.64(1.69)$ & -3.52 & $0.002^{* *}$ \\
45 min & $28.67(1.96)$ & $30.58(2.49)$ & -2.39 & $0.002^{* *}$ \\
60 min & $59.34(4.36)$ & $58.74(4.27)$ & 0.37 & 0.71 \\
120 min & $122.82(7.29)$ & $117.67(6.85)$ & 1.90 & 0.07 \\
240 min & $950.42(25.96)$ & $959.74(27.53)$ & -0.75 & 0.46 \\
1,440 min & & & & \\
\hline
\end{tabular}

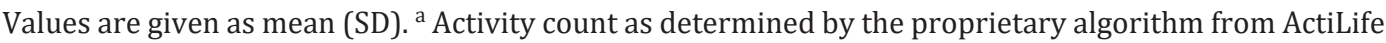
software. ${ }^{*} p<0.05 ;{ }^{* *} p<0.005$.

noticeable in the 30 -min period following waking $(t=-3.24, p=0.0002)$. Table 4 depicts the differences between groups for the PRO data, reported as means across the full 28 days of testing. Significant differences in pain and stiffness were noted between the groups $(p<$ 0.005). No correlation between PROs and activity counts were seen. No clear relationship between activity counts and PROs was seen.

\section{Discussion/Conclusion}

This study is the first to show that a detailed analysis of actigraphy variables could identify activity pattern changes associated with arthritis, where the daily summaries did not. These results suggest that the use of discrete variables may be a useful tool to help identify clinical cohorts and, thus, should be explored further to determine if they may be used as an effective clinical biomarker.

Despite the ubiquitous presence of wearable sensors, limited research exists that directly compares the activity patterns of healthy adults and arthritis patients over sustained periods of time, while no other study has yet considered whether this data can be explored further to identify meaningful digital biomarkers. Although a recent study using mobile phones to identify biomarkers shows promise, it focused on specific, short-duration walking tasks rather than general PA, and was limited by its low success rate in meeting data collection quality criteria [8]. In studies that have investigated general PA patterns, arthritis patients have been shown to move significantly less than their healthy counterparts $[15,16]$, a result not replicated in this study. However, key differences exist which may partially explain these discrepancies. First, previous studies typically evaluated activity over a shorter duration of time, ranging from $<1$ week [16] to 14 days [10], or focused on self-report measures of activity [27]. More habitual and normal patterns of activity may emerge with time; however, as 


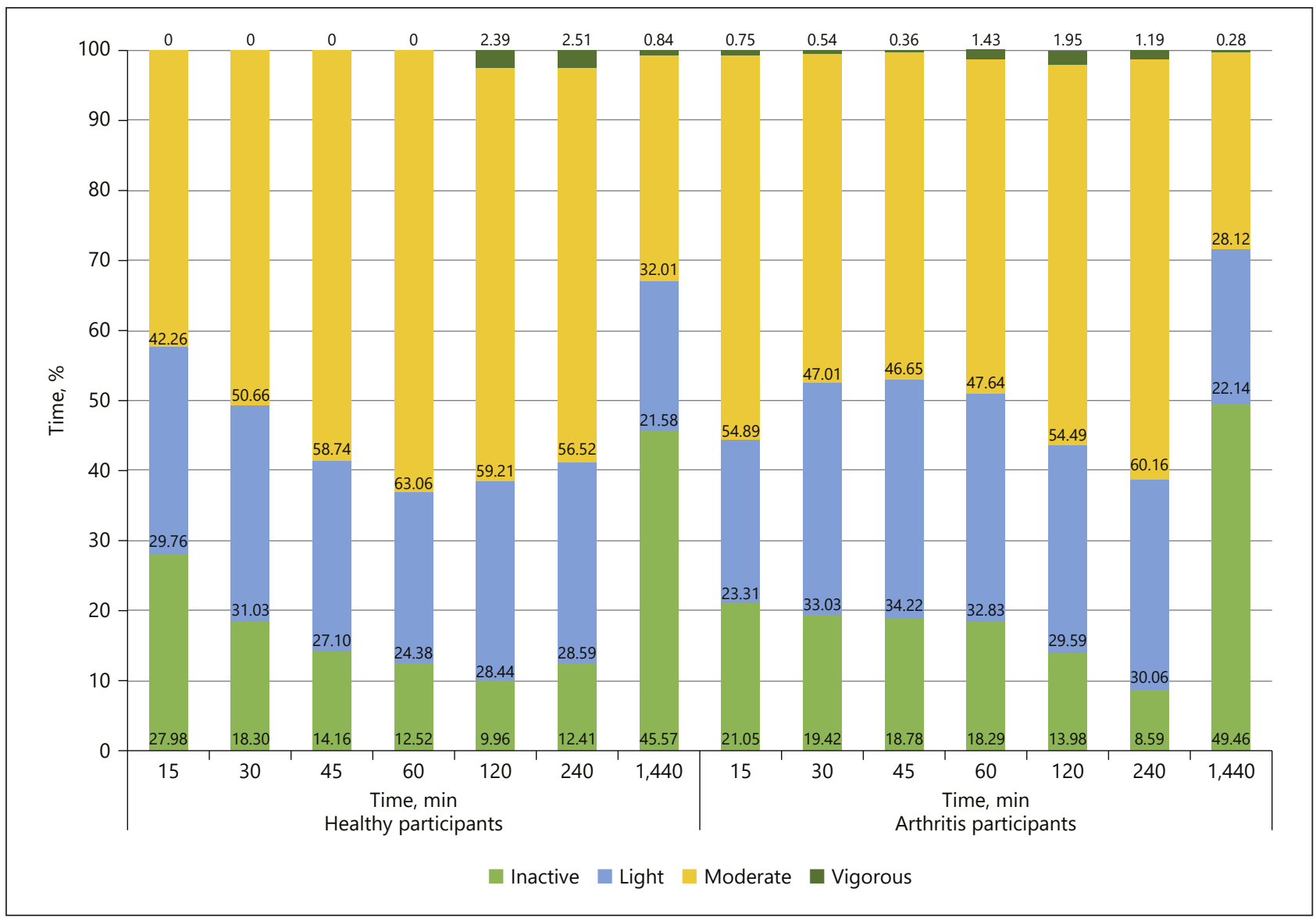

Fig. 1. The percentage of time participants spent in each activity threshold, according to the daily aggregate activity counts. Activity count thresholds: $0-1,800$, inactive; 1,801-2,690, light; 2,691-6,166, moderate; $>6,166$, vigorous.

Fig. 2. AUC analysis for activity counts in the first 30 min following waking, demonstrating a front-loaded pattern of activity in arthritis patients compared to controls.

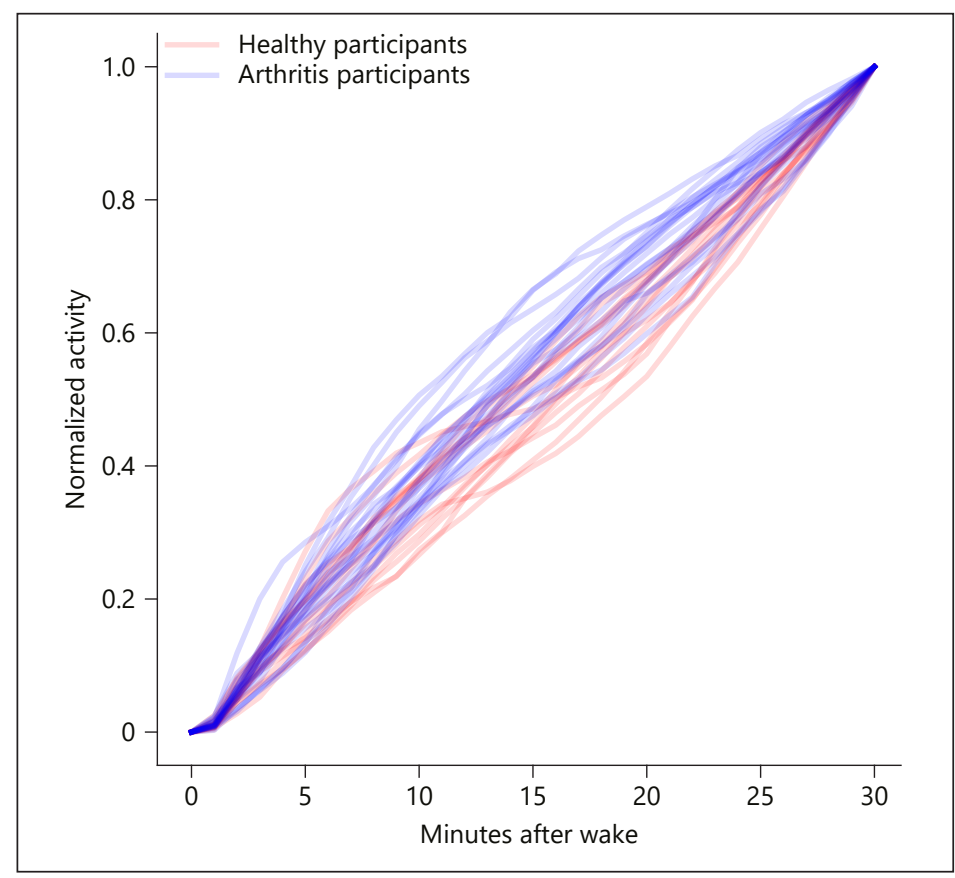


Table 4. Differences in patient-reported outcomes (PRO) between healthy controls and arthritis patients, reported as mean values across the full 28 days of testing

\begin{tabular}{|c|c|c|c|c|}
\hline Dependant variable & $\begin{array}{l}\text { Healthy } \\
\text { controls }\end{array}$ & $\begin{array}{l}\text { Arthritis } \\
\text { patients }\end{array}$ & $t$ test & $p$ value \\
\hline \multicolumn{5}{|l|}{$\operatorname{Pain}^{a}$} \\
\hline In the last $24 \mathrm{~h}$, reported in the evening & $0.33(0.82)$ & $3.64(2.8)$ & 25.56 & $<0.0005$ \\
\hline During the day, reported in the evening & $0.29(0.60)$ & $3.54(2.74)$ & 26.20 & $<0.0005$ \\
\hline Overnight, reported in the morning & $0.24(0.68)$ & $3.17(2.62)$ & 25.36 & $<0.0005$ \\
\hline In the morning, reported upon waking & $0.32(0.71)$ & $3.32(2.65)$ & 26.64 & $<0.0005$ \\
\hline \multicolumn{5}{|l|}{ Stiffness ${ }^{b}$} \\
\hline Duration of stiffness (maximum number of minutes) & $27.27(42.34)$ & $233.68(468.68)$ & 9.35 & $<0.0005$ \\
\hline $30 \min (\%$ of participants) & 0.0 & 0.4 & & \\
\hline $30 \mathrm{~min}$ to $1 \mathrm{~h}$ ( $\%$ of participants) & 0.0 & 0.0 & & \\
\hline $1-2 \mathrm{~h}$ (\% of participants) & 21.2 & 22.6 & & \\
\hline $2-4 \mathrm{~h}$ ( $\%$ of participants) & 12.1 & 19.6 & & \\
\hline $4 \mathrm{~h}$ (\% of participants) & 0.0 & 4.5 & & \\
\hline All day (\% of participants) & 0.0 & 12.9 & & \\
\hline None (\% of participants) & 66.7 & 40.0 & & \\
\hline Severity of stiffness during the day, reported in the evening & $0.33(0.52)$ & $1.73(0.94)$ & 27.56 & $<0.0005$ \\
\hline Intensity of stiffness in the morning, reported upon waking & $0.41(0.56)$ & $1.66(0.56)$ & 25.60 & $<0.0005$ \\
\hline
\end{tabular}

Values are given as mean (SD) unless indicated otherwise. ${ }^{\text {a }}$ Pain was reported on a 10 -point Likert scale, where $0=$ no pain; ${ }^{\mathrm{b}}$ stiffness was rated on a scale of $0-4$ with severity increasing with the score.

baseline activity was not controlled for within this study, it is unclear whether this was the case in this cohort. Furthermore, previous research typically focused on a single arthritic cohort (e.g., specifically rheumatoid arthritis), rather than the variety of arthritic conditions evaluated in this study. Additionally, the cohort tested in this study was younger [15], was classed as lightly or moderately active [10], while the majority were all classified as being stable or with low disease activity [10], when compared to previous research. It is possible that participants in this study were less symptomatic or were more likely to still be of working age and therefore may be expected to have similar movement patterns during the day, irrespective of any symptoms they may be experiencing.

Due to the variability in how daily activity may be reported or measured, the purpose of this study was to determine whether more discrete measures of activity were able to distinguish between healthy and clinical cohorts as the summary-level data does not explain how people move, but simply whether they move or not. In this way, the exploratory results from this study are potentially significant, as they highlight there is value in diving deeper into the data to understand how conditions such as arthritis may influence behaviour. In particular, it was decided to focus on activities following waking as this is an area that may often be symptomatic within arthritis patients. The assessment of the AUC for these discrete variables highlighted some statistically significant differences between the two cohorts. Interestingly, arthritis patients demonstrated higher activity counts, compared to their healthy counterparts within the early hours of waking. This difference was particularly noticeable in the first $30 \mathrm{~min}$ following waking. This is in contrast to previously published research [10]. However, previous research reported differences in sedentary time across the day between healthy and arthritic participants, who were all classified as sedentary. In contrast, this study has demonstrated that although in each examined time period both cohorts move the same amount, the manner in which they distribute their activity over time differs. Specifically, arthritis patients move more, earlier in the examined time periods. This initially appears counter-intuitive as 
morning stiffness is a common symptom in arthritis, and so it would be expected that such stiffness would limit this observed greater movement early in the morning, especially within the first hour [22]. Indeed, qualitative research has suggested that morning stiffness severely impacts functional tasks including work [22]. For instance, an antalgic gait or short cadence due to pain or stiffness may result in greater steps in the morning until symptoms ease.

However, functional limitations typically correlate with the severity of self-reported symptoms [20,28], yet no correlations were seen between PROs and activity accounts in this study, despite the differences in PROs between the cohorts. This suggests that this early morning difference in activity patterns may be a habitual coping strategy. It is possible that arthritis patients, through lived experiences of their condition, know that they need to react to their symptoms whether positive or negative. For example, if participants feel well upon waking, they may try to ensure that they start their tasks and functional activities early due to the variable nature of the condition [21,29], in order to get ahead of the condition. Alternatively, participants with high stiffness may attempt to do more than usual earlier in the morning as they are aware of the benefits that being active has on their symptoms [21,29-31]. Finally, it may be that the day-to-day fluctuations in self-reported pain simply do not affect the way people are organizing their early morning activities, and that participants with arthritis know that they need to "go on as usual" and thus will work against the stiffness or other morning symptoms in order to ensure that they are up and functioning as much as they can so as to allow them continue with the rest of their day [29, 32, 33]. Nevertheless, it cannot be excluded that in the presence of significant variations in pain/stiffness, the early-morning activity would be significantly affected.

It is clear from the literature that participants with arthritic conditions will use a variety of strategies to help them cope with their condition [26-30], and it may be expected that these strategies may be evident in behaviours such as their movement patterns. Previously, the subtlety of these strategies may not have been obvious in summary-level activity data. Thus, it is likely that the strategies employed by participants are manifested by how they move, rather than whether they move, thus subtle differences require subtle assessments in order to accurately capture them and, by association, understand them.

Irrespective of whether participants were reacting to increased or decreased symptoms, this study has highlighted that in the morning they appear to distribute their movements differently to healthy controls. A main strength of this study is that participants were monitored over a 28-day period, thus we can be confident that the findings are reflective of their normal behaviours. However, as this was an exploratory study, no power calculation had been performed. Thus, future work needs to evaluate these patterns within a large cohort to confirm its presence further. Furthermore, baseline activity and disease severity were not controlled for within this analysis. Nonetheless, this study has demonstrated the potential usefulness of monitoring discrete-level data to compare and differentiate clinical and healthy cohorts. With further investigation, this may help identify meaningful behavioural digital biomarkers that can be used in clinical trials to establish intervention effectiveness.

\section{Statement of Ethics}

The study protocol was approved by the research institute's Committee on Human Research. The participants of this study gave their written informed consent prior to participation. Ethical approval was obtained for this study for all participants - for the patients through Trinity College Dublin/Tallaght Hospital and for the healthy volunteers through University College Dublin. The informed consent allows Novartis Pharma AG to share the data from this study with direct collaborators only. Collaboration proposals are welcome.

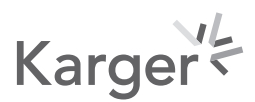




\section{Disclosure Statement}

The authors have no conflicts of interest to declare. J.F.D., V.I., and F.C. are employees of Novartis Pharma AG, Basel, Switzerland.

\section{Funding Sources}

This study was funded by Novartis Pharma AG, Basel, Switzerland.

\section{Author Contributions}

A.K. prepared the manuscript for publication. N.S., V.I., and B.M. completed the statistical analysis of the data. B.M., J.F.D., F.C., and B.C. contributed to the conception and design of the study. S.D., R.M., D.G., M.M.-D. were involved in the recruitment or participants and project management of participants. All authors reviewed the final version for publication and agree to be accountable for all aspects of this work.

\section{References}

1 Woolf AD. Global burden of osteoarthritis and musculoskeletal diseases. BMC Musculoskelet Disord. 2015; 16(Suppl 1):S3.

2 Coravos A, Khozin S, Mandl KD. Developing and adopting safe and effective digital biomarkers to improve patient outcomes. NPJ Digit Med. 2019;2(1):14.

3 Del Din S, Godfrey A, Mazzà C, Lord S, Rochester L. Free-living monitoring of Parkinson's disease: lessons from the field. Mov Disord. 2016 Sep;31(9):1293-313.

4 Guthrie NL, Carpenter J, Edwards KL, Appelbaum KJ, Dey S, Eisenberg DM, etal. Emergence of digital biomarkers to predict and modify treatment efficacy: machine learning study. BMJ Open. 2019 Jul;9(7):e030710.

5 Gold M, Amatniek J, Carrillo MC, Cedarbaum JM, Hendrix JA, Miller BB, et al. Digital technologies as biomarkers, clinical outcomes assessment, and recruitment tools in Alzheimer's disease clinical trials. Alzheimers Dement (N Y). 2018 May;4(1):234-42.

6 FDA. Patient focused drug development: collecting comprehensive and representative input. USA: U.S. Department of Health and Human Services Food and Drug Administration; 2018.

7 Torous J, Rodriguez J, Powell A. The New Digital Divide For Digital BioMarkers. Digit Biomark. 2017 Sep;1(1): 87-91.

8 Hamy V, Garcia-Gancedo L, Pollard A, Myatt A, Liu J, Howland A, et al. Developing Smartphone-Based Objective Assessments of Physical Function in Rheumatoid Arthritis Patients: the PARADE Study. Digit Biomark. 2020 Apr;4(1):26-43.

9 Ainsworth B, Cahalin L, Buman M, Ross R. The current state of physical activity assessment tools. Prog Cardiovasc Dis. 2015 Jan-Feb;57(4):387-95.

10 Prioreschi A, Hodkinson B, Avidon I, Tikly M, McVeigh JA. The clinical utility of accelerometry in patients with rheumatoid arthritis. Rheumatology (Oxford). 2013 Sep;52(9):1721-7.

11 Reeder B, David A. Health at hand: A systematic review of smart watch uses for health and wellness. J Biomed Inform. 2016 Oct;63:269-76.

12 Phillips SM, Cadmus-Bertram L, Rosenberg D, Buman MP, Lynch BM. Wearable Technology and Physical Activity in Chronic Disease: opportunities and Challenges. Am J Prev Med. 2018 Jan;54(1):144-50.

13 Troiano RP, McClain JJ, Brychta RJ, Chen KY. Evolution of accelerometer methods for physical activity research. Br J Sports Med. 2014 Jul;48(13):1019-23.

14 Dunlop DD, Song J, Semanik PA, Chang RW, Sharma L, Bathon JM, et al. Objective physical activity measurement in the osteoarthritis initiative: are guidelines being met? Arthritis Rheum. 2011 Nov;63(11):3372-82.

15 Hashimoto T, Yoshiuchi K, Inada S, Shirakura K, Wada N, Takeuchi K, et al. Physical activity of elderly patients with rheumatoid arthritis and healthy individuals: an actigraphy study. Biopsychosoc Med. 2015 Oct;9(1):19.

16 Murphy SL, Smith DM, Clauw DJ, Alexander NB. The impact of momentary pain and fatigue on physical activity in women with osteoarthritis. Arthritis Rheum. 2008 Jun;59(6):849-56.

17 Summers G, Booth A, Brooke-Wavell K, Barami T, Clemes S. Physical activity and sedentary behavior in women with rheumatoid arthritis: a comparison of patients with low and high disease activity and healthy controls. Open Access Rheumatol. 2019 Jun;11:133-42.

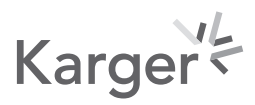


18 van Genderen S, Boonen A, van der Heijde D, Heuft L, Luime J, Spoorenberg A, et al. Accelerometer quantification of physical activity and activity patterns in patients with anklyosing spondylitis and population controls. J Rheumatol. 2015 Dec;42(12):2369-75.

19 Tomkins-Lane C, Norden J, Sinha A, Hu R, Smuck M. Digital biomarkers of spine and musculoskeletal disease from accelerometers: defining phenotypes of free-living physical activity in knee osteoarthritis and lumbar spinal stenosis. Spine J. 2019 Jan;19(1):15-23.

20 Gignac MA, Davis AM, Hawker G, Wright JG, Mahomed N, Fortin PR, et al. "What do you expect? You're just getting older": A comparison of perceived osteoarthritis-related and aging-related health experiences in middle- and older-age adults. Arthritis Rheum. 2006 Dec;55(6):905-12.

21 Halls S, Dures E, Kirwan J, Pollock J, Baker G, Edmunds A, et al. Stiffness is more than just duration and severity: a qualitative exploration in people with rheumatoid arthritis. Rheumatology (Oxford). 2015 Apr;54(4):61522.

22 Phillips S, Dow L. Impact of impaired morning function on quality of life in rheumatoid arthritis results of an exploratory patient survey. Int J Clin Rheumatol. 2012;7(6):597-606.

23 Jacquemin C, Servy H, Molto A, Sellam J, Foltz V, Gandjbakhch F, et al. Physical activity assessment using an activity tracker in patients with rheumatoid arthritis and axial spondyloarthritis: prospective observational study. JMIR Mhealth Uhealth. 2018 Jan;6(1):e1.

24 Perraudin CG, Illiano VP, Calvo F, O’Hare E, Donnelly SC, Mullan RH, et al. Observational study of a wearable sensor and smartphone application supporting unsupervised exercises to assess pain and stiffness. Digit Biomark. 2018 Oct;2(3):106-25.

25 Sett N, Mac Namee B, Calvo F, Caulfield B, Costello J, Donnelly S, et al. Are you in pain? Predicting pain and stiffness from wearable sensor activity data. SGAI International Conference on Artificial Intelligence; 17-19 December 2019; Cambridge, England. 2019. https://doi.org/10.1007/978-3-030-34885-4_15.

26 van Hees VT, Fang Z, Langford J, Assah F, Mohammad A, da Silva IC, et al. Autocalibration of accelerometer data for free-living physical activity assessment using local gravity and temperature: an evaluation on four continents. J Appl Physiol (1985). 2014 Oct;117(7):738-44.

27 Henchoz Y, Bastardot F, Guessous I, Theler JM, Dudler J, Vollenweider P, et al. Physical activity and energy expenditure in rheumatoid arthritis patients and matched controls. Rheumatology (Oxford). 2012 Aug;51(8): 1500-7.

28 Gossec L, Guyard F, Leroy D, Lafargue T, Seiler M, Jacquemin C, et al. Detection of flares by decrease in physical activity, collected using wearable activity trackers in rheumatoid arthritis or axial spondyloarthritis: an application of machine learning analyses in rheumatology. Arthritis Care Res (Hoboken). 2019 Oct; 71(10):133643.

29 Hendry M, Williams NH, Markland D, Wilkinson C, Maddison P. Why should we exercise when our knees hurt? A qualitative study of primary care patients with osteoarthritis of the knee. Fam Pract. 2006 Oct;23(5):55867.

30 Orbai AM, Smith KC, Bartlett SJ, De Leon E, Bingham CO 3rd. "Stiffness has different meanings, I think, to everyone": examining stiffness from the perspective of people living with rheumatoid arthritis. Arthritis Care Res (Hoboken). 2014 Nov;66(11):1662-72.

31 Wilcox S, Der Ananian C, Abbott J, Vrazel J, Ramsey C, Sharpe PA, et al. Perceived exercise barriers, enablers, and benefits among exercising and nonexercising adults with arthritis: results from a qualitative study. Arthritis Rheum. 2006 Aug;55(4):616-27.

32 Grønning K, Lomundal B, Koksvik HS, Steinsbekk A. Coping with arthritis is experienced as a dynamic balancing process. A qualitative study. Clin Rheumatol. 2011 Nov;30(11):1425-32.

33 Kanavaki AM, Rushton A, Efstathiou N, Alrushud A, Klocke R, Abhishek A, et al. Barriers and facilitators of physical activity in knee and hip osteoarthritis: a systematic review of qualitative evidence. BMJ Open. 2017 Dec;7(12):e017042. 